\title{
Utilizing personalized stereotactic frames for laser interstitial thermal ablation of posterior fossa and mesiotemporal brain lesions: a single-institution series
}

\author{
David Y. A. Dadey, PhD, ${ }^{1,2}$ Ashwin A. Kamath, MD, ${ }^{1}$ Matthew D. Smyth, MD,1 \\ Michael R. Chicoine, MD, ${ }^{1}$ Eric C. Leuthardt, MD, ${ }^{1}$ and Albert H. Kim, MD, PhD ${ }^{1}$ \\ 'Department of Neurological Surgery, and ${ }^{2}$ Medical Scientist Training Program, Washington University School of Medicine, \\ St. Louis, Missouri
}

\begin{abstract}
OBJECTIVE The precision of laser probe insertion for interstitial thermal therapy of deep-seated lesions is limited by the method of stereotactic guidance. The objective of this study was to evaluate the feasibility of customized STarFix 3Dprinted stereotactic platforms to guide laser probe insertion into mesiotemporal and posterior fossa targets.
\end{abstract}

METHODS The authors conducted a retrospective review of 5 patients (12-55 years of age) treated with laser interstitial thermal therapy (LITT) in which STarFix platforms were used for probe insertion. Bone fiducials were implanted in each patient's skull, and subsequent CT scans were used to guide the design of each platform and incorporate desired treatment trajectories. Once generated, the platforms were mounted on the patients' craniums and used to position the laser probe during surgery. Placement of the laser probe and the LITT procedure were monitored with intraoperative MRI. Perioperative and follow-up MRI were performed to identify and monitor changes in target lesions.

RESULTS Accurate placement of the laser probe was observed in all cases. For all patients, thermal ablation was accomplished without intraoperative complications. Of the 4 patients with symptomatic lesions, 2 experienced complete resolution of symptoms, and 1 reported improved symptoms compared with baseline.

CONCLUSIONS Customized stereotactic platforms were seamlessly incorporated into the authors' previously established LITT workflow and allowed for accurate treatment delivery.

http://thejns.org/doi/abs/10.3171/2016.7.FOCUS16207

KEY WORDS laser interstitial thermal therapy; LITT; STarFix; stereotactic guidance; 3D-printed frame; brain tumor

$\mathrm{T}$ HE treatment of brain neoplasms and structural anomalies associated with epilepsy relies on multimodal therapy coordinated by neurosurgeons, neurologists, medical oncologists, and radiation oncologists. Despite extensive developments in the technologies and techniques available to neurosurgeons, the location of the intracranial lesion plays a significant role in determining the approach and overall feasibility of a surgical procedure. Specifically, lesions deep within the brain or near eloquent areas are often difficult to access surgically due to risks posed to neurological function. Consistent with these established neurosurgical principles, a recent study by Ferroli et al. quantified surgical risk and identified the posterior fossa location and involvement of eloquent brain regions as 2 of 5 factors associated with poorer outcomes after brain tumor excision. ${ }^{3}$

With the advent of laser interstitial thermal therapy (LITT), deep-seated lesions, which traditionally required craniotomies and development of a surgical corridor through normal brain parenchyma, can now be treated with a minimally invasive approach. Several lesion types including epileptogenic foci, tumors, and radiation necrosis have been shown to respond to thermal ablation. ${ }^{5,6,15,17}$ Furthermore, LITT has benefited greatly from intraoperative MRI, which allows for real-time monitoring of thermal ablation to ensure conformality of treatment zones. ${ }^{16}$

ABBREVIATIONS DBS = deep brain stimulation; EEG = electroencephalography; ICU = intensive care unit; LITT = laser interstitial thermal therapy; $\mathrm{mT}=$ microTargeting; TDT $=$ thermal damage threshold. 


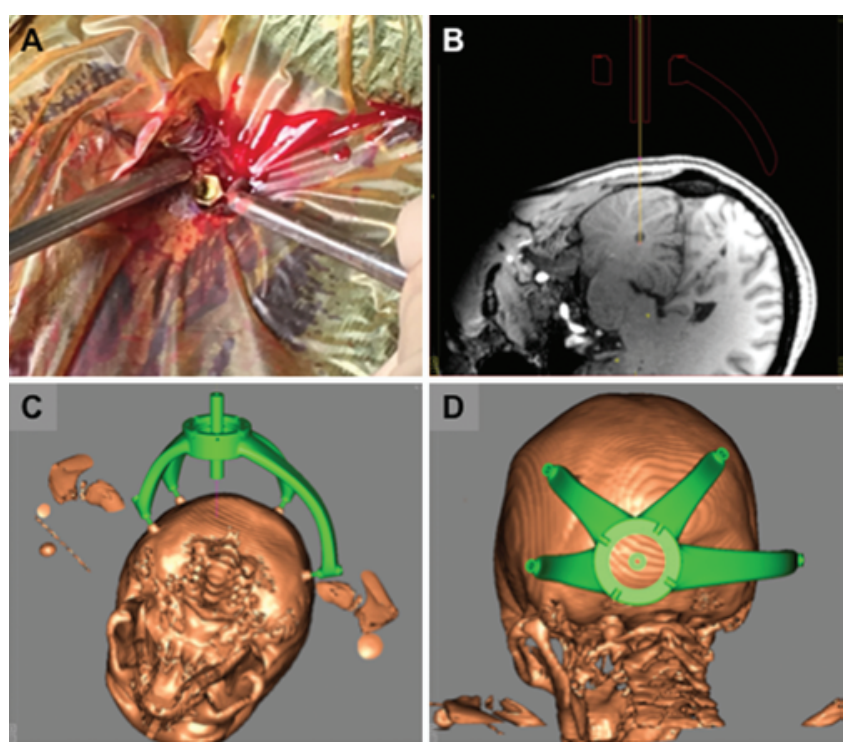

FIG. 1. Design of the mT platform (Case 5). A: Image of a bone fiducial (gold) after placement, which was used for CT coregistration of skull anatomy with a rendered $3 \mathrm{D}$ model of the $\mathrm{mT}$ platform. B: Desired probe trajectory. $\mathbf{C}$ and $\mathbf{D}$ : Images from planning software showing the predicted structure of the $\mathrm{mT}$ platform (green).

However, the accuracy of ablative procedures may be limited by variations in the stability of the stereotactic guidance platform. In particular, tumors in the posterior fossa-within the cerebellum or brainstem-and lesions of the mesiotemporal lobes require exquisitely precise targeting to effect appropriate treatment and simultaneously spare adjacent critical tissues. Additionally, the difficulty of accurately placing laser probes into such targets is compounded by errors in coregistration. Frameless methods, which rely on fiducial stickers and tracing of surface anatomy, are subject to limitations posed by the variability of mobile scalp tissue. In addition, biopsy/laser probe bases are variably stable enough to withstand the force of drills passing through them at oblique angles to the skull.

The STarFix microTargeting (mT) platform (FHC Inc.) offers a solution to the problem of accurate laser probe insertion, particularly for posterior fossa and medial temporal lobe targets. By using bone fiducials (Fig. 1A) and rapid-prototyping 3D printing technology, STarFix mT platforms can be designed and manufactured to fit the unique cranial anatomy of each patient. Bone fiducials, by serving as fixed anchor points, may allow for the planning of highly accurate and stable treatment trajectories. ${ }^{19}$ STarFix $\mathrm{mT}$ platforms have been successfully used for deep brain stimulation (DBS) ${ }^{9}$ and for epilepsy monitoring:,1,18 however, to date no studies have examined their application to LITT. In this paper we present a series of 5 patients who presented with intracranial lesions that were amenable to LITT with STarFix stereotactic guidance.

\section{Methods}

\section{Study Design and Patient Selection}

This was a retrospective single-institution review of 5 patients with epilepsy or brain tumors in the posterior fossa or mesiotemporal regions. Approval for this study
TABLE 1. Patient demographics and preoperative data

\begin{tabular}{cccl}
\hline Case No. & Age (yrs), Sex & Lesion & \multicolumn{1}{c}{ Location } \\
\hline 1 & $25, \mathrm{~F}$ & Epilepsy focus & Rt basal temporal \\
\hline 2 & $55, \mathrm{M}$ & Ganglioglioma & Rt dorsal pons \\
\hline 3 & $17, \mathrm{~F}$ & Epilepsy focus & Lt mesiotemporal \\
\hline 4 & $12, \mathrm{~F}$ & Epilepsy focus & Lt mesiotemporal \\
\hline 5 & $36, \mathrm{~F}$ & Ganglioglioma & Lt medial cerebellum \\
\hline
\end{tabular}

was obtained from the Washington University Institutional Review Board. Information was obtained from clinical records, operative notes, and radiological data. Patients were selected for the NeuroBlate System (Monteris Medical Corporation) by the senior authors (E.C.L. and A.H.K), and were treated at Barnes-Jewish Hospital (Washington University, St. Louis, Missouri) from May 2014 to February 2016. Evaluation of patients with brain tumors involved a multidisciplinary tumor board consisting of neurosurgeons, neurologists, medical oncologists, and radiation oncologists. Patients were ultimately considered candidates for LITT based on the clinical judgment of both senior authors. Evaluation of patients with epilepsy involved a multidisciplinary effort (epilepsy conference) by a team of neurosurgeons, neurologists, and radiologists. For Cases 1, 3, and 4, epileptogenic foci were considered for LITT only after a corresponding lesion could be delineated with invasive electroencephalography (EEG) video monitoring and FDG/PET or ictal SPECT.

\section{Patient Characteristics}

This study included 5 patients (4 females and 1 male, ages $12-55$ years old). Of the lesions treated, 3 were epileptogenic foci and 2 were glial neoplasms (Table 1). All patients were receiving surgical treatment for the first time. Patient 2 had undergone needle biopsy of the pontine lesion prior to his LITT procedure. Patient 5 underwent stereotactic biopsy as part of the LITT procedure.

\section{STarFix LITT Procedure}

To allow for custom production of the STarFix mT platform for each patient, the surgical procedure was performed in 2 phases. The first phase of the procedure involved placement of bone fiducials, which would serve as anchors for the STarFix frame. Four fiducials were placed in a patient under general anesthesia through separate $1-\mathrm{cm}$ incisions, and the skin and soft tissue closed in 1 layer using Monocryl 3-0 suture (Fig. 1A). Patients were scanned postoperatively using thin-slice CT and left the hospital the day of surgery. Trajectory planning and the design of each frame were completed in Waypoint Navigator Software (FHC Inc.) using coregistered CT and MRI (Fig. 1BD). Following the completion of surgical planning, the $\mathrm{mT}$ frame was constructed by the manufacturer by 3D printing within 4 days, sterilized in our institution over 1 day, and then was ready for mounting typically within 1 week.

For the second phase of the procedure (1 week later), an MRI-compatible head holder was placed on patients while under general anesthesia, and the Medtronic StealthStation MRI-guided navigation system was coregistered with pre- 
TABLE 2. LITT volume and dose parameters

\begin{tabular}{cccccr}
\hline $\begin{array}{c}\text { Case } \\
\text { No. }\end{array}$ & $\begin{array}{c}\text { Target } \\
\text { Vol } \\
\left(\mathrm{cm}^{3}\right)\end{array}$ & $\begin{array}{c}\text { Yellow } \\
\text { TDT Line } \\
\text { Vol }\left(\mathrm{cm}^{3}\right)\end{array}$ & $\begin{array}{c}\% \\
\text { Treated to } \\
\text { Yellow }\end{array}$ & $\begin{array}{c}\text { Blue* } \\
\text { TDT Line } \\
\text { Vol }\left(\mathrm{cm}^{3}\right)\end{array}$ & $\begin{array}{c}\% \\
\text { Treated to } \\
\text { Blue }\end{array}$ \\
\hline 1 & NA & 31.1 & 100.0 & 24.2 & 100.0 \\
\hline 2 & 17.0 & 18.4 & 75.5 & 13.6 & 62.2 \\
\hline 3 & NA & 12.0 & 100.0 & 8.3 & 100.0 \\
\hline 4 & 5.4 & 14.4 & 86.6 & 10.3 & 79.2 \\
\hline 5 & 8.7 & 23.8 & 100.0 & 19.4 & 100.0 \\
\hline NA = not applicable. \\
* Yellow was heated to the equivalent of $43^{\circ} \mathrm{C}$ for 2 minutes; blue was heated \\
to the equivalent of $43^{\circ} \mathrm{C}$ for 10 minutes.
\end{tabular}

operative MRI scans. The fiducial incisions were opened, and the STarFix mT frame was mounted and secured on the bone fiducials. The NeuroBlate System was then combined with the $\mathrm{mT}$ frame, which was used to guide and stabilize the laser probe driver. We typically perform a 5 -mm stab incision followed by a 4.5 - $\mathrm{mm}$ bur hole for insertion of the laser probe. With the probe inserted in the lesion, STEALTH navigation was used to confirm the accuracy of the planned trajectory, and an IMRIS intraoperative 1.5-T MRI machine was used to monitor thermal ablation using real-time MR thermometry. Thermal damage threshold (TDT) lines were used to guide treatment time and conformality of the treatment zone (Table 2). The TDT lines are yellow and blue lines that encircle regions of tissue heated to the equivalent of $43^{\circ} \mathrm{C}$ for 2 minutes and $43^{\circ} \mathrm{C}$ for 10 minutes, respectively. After completion of the ablation procedure, patients were observed in the intensive care unit (ICU) prior to discharge. The length of ICU and total hospital stay ranged from 1 to 2 days, and 1 to 3 days, respectively.

\section{Results}

STarFix mT platforms were used to treat 5 patients with LITT. In all cases, the platforms were successfully designed and mounted on the implanted bone fiducials. Probe insertion into the target lesions was confirmed with intraoperative MRI, and delivery of LITT was accomplished without complication. Mean total operative time, combining fiducial placement and laser ablation surgeries, was 3 hours and 32 minutes \pm 53 minutes (Table 3 ). Patients 1 and 3, who presented for ablation of epileptogenic foci, experienced resolution of seizure symptoms with no postoperative neurological deficits. Patient 4 , another patient with epilepsy, did not respond to therapy. Patient 2 , who presented with a brainstem ganglioglioma, experienced improvement in preoperative sensory symptoms after a postoperative period of transient ophthalmological and speech deficit. The cerebellar ganglioglioma of patient 5 was ablated with no postoperative symptoms.

\section{Case 1}

A 25-year-old woman presented with an extensive history of complex partial seizures defined by staring spells and heavy breathing with associated auras. Using video EEG with ictal SPECT, a right posterior temporal seizure
TABLE 3. Operative time

\begin{tabular}{cccc}
\hline Case No. & Phase 1* $^{\text {(hrs:min) }}$ & Phase $2 \dagger$ (hrs:min) & Total (hrs:min) \\
\hline 1 & $0: 43$ & $3: 59$ & $4: 42$ \\
\hline 2 & $0: 29$ & $2: 34$ & $3: 03$ \\
\hline 3 & $0: 15$ & $3: 54$ & $4: 09$ \\
\hline 4 & $0: 12$ & $3: 03$ & $3: 15$ \\
\hline 5 & $0: 21$ & $2: 08$ & $2: 29$ \\
\hline Mean & $0: 24$ & $3: 08$ & $3: 32$ \\
\hline
\end{tabular}

* Phase 1 is the first operation for placement of the bone fiducials.

$\dagger$ Phase 2 is the second operation for the LITT procedure.

focus with right posterior temporal discharge was detected. Further characterization with MRI revealed right lateral periventricular heterotopia (Fig. 2A) consistent with the findings on ictal SPECT. The patient was offered lesionectomy with selective amygdalohippocampectomy or laser ablation, and opted to receive laser ablation of this right temporal lesion as a treatment for her epilepsy. Following placement of bone fiducials and assembly of her STarFix frame, laser therapy was delivered along an anterior to posterior vector to a tubular treatment zone approximately $3 \mathrm{~cm}$ in diameter and $5 \mathrm{~cm}$ in length (Fig. 2D). Treatment was completed without complication, and the patient was discharged the following day after observation in the ICU. She was seen for follow-up at 10 days, 3 months, and 9 months after surgery and reported no new seizures. No neurological deficits were found in her follow-up examinations, and she remains Engel Class I. Follow-up MRI at 3 months showed a persistent T1-weighted Gd-enhancing signal consistent with successful laser ablation of the medial posterior right temporal lobe (Fig. 2C), with mildly increased $\mathrm{Gd}$ enhancement of ablated structures relative to immediate postoperative imaging (Fig. 2B).

\section{Case 2}

A 55-year-old man presented to his primary care physician with numbness in his left fingers and toes. Brain MRI revealed a T1 hypointense, T2 hyperintense, $2.1 \times 2.3 \times 2$ $\mathrm{cm}$ mass in the pons (Fig. 3A). Stereotactic needle biopsy identified the mass as a WHO Grade I ganglioglioma. Progressive symptoms of numbness and paresthesias of the face led him to seek therapy. Given the size and location of the lesion, the therapeutic goal was to reduce the tumor burden by laser ablation of the central core. During surgery, the laser probe was successfully inserted into the core of the tumor (Fig. 3D and E), and laser treatment was delivered to $80 \%-90 \%$ of the tumor within the tumor center. Postoperatively (Fig. 3B) in the ICU, the patient presented with internuclear ophthalmoplegia, right eye ophthalmoplegia, dysarthria, and reduced sensation on his left side. $\mathrm{He}$ was treated with dexamethasone and monitored for 2 days, after which he was transferred to the stepdown unit. He was discharged to a rehabilitation center per physical/ occupational therapy recommendations, and his symptoms gradually improved on subsequent follow-up. Six months after surgery (Fig. 3C), his ophthalmoplegia had resolved completely, and he reported improved speech and general function relative to his baseline before surgery. 

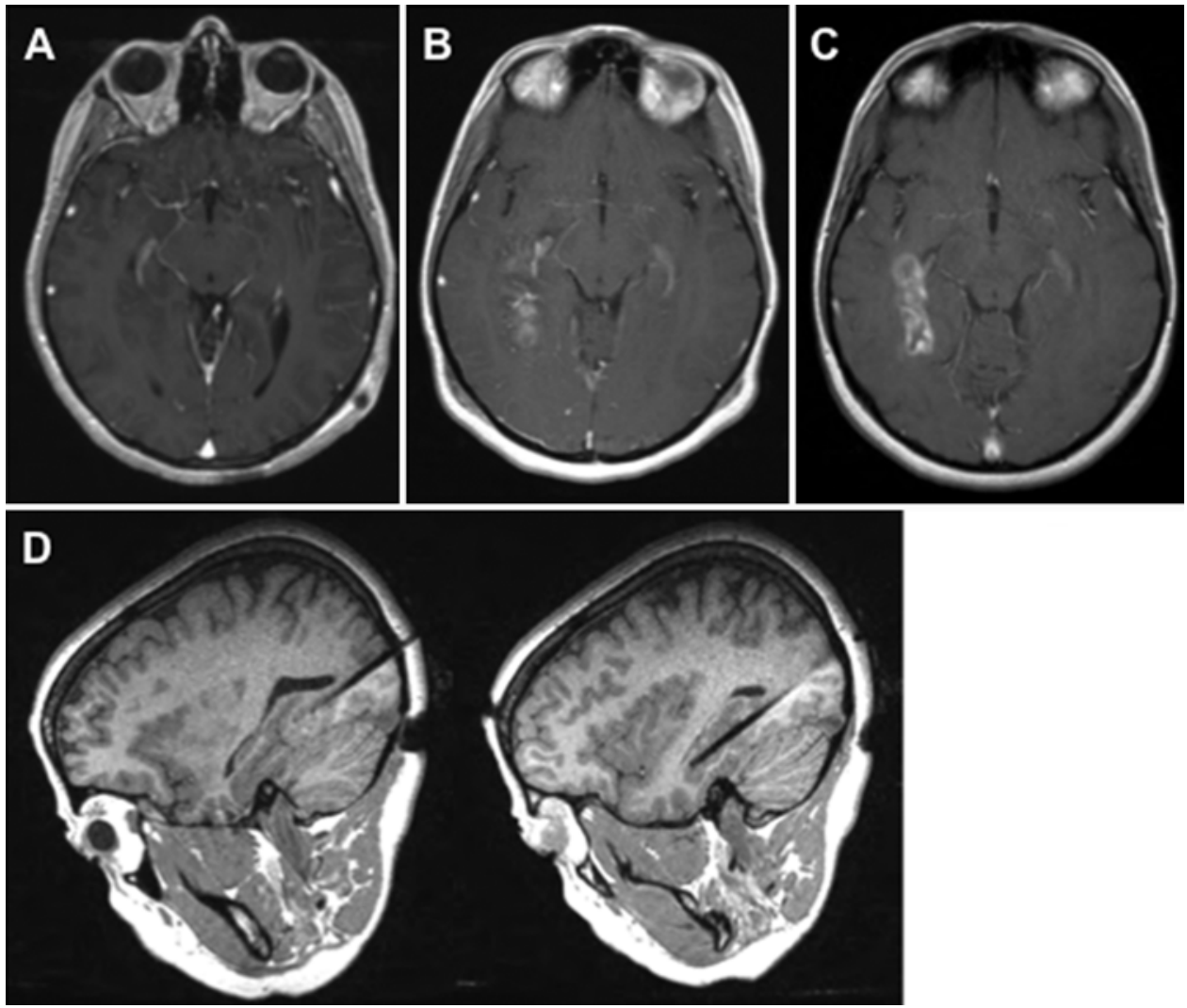

FIG. 2. Epilepsy focus. Magnetic resonance imaging of Case 1 showing a right basal temporal lesion treated with LITT. A: Preoperative axial T1-weighted MR image with Gd contrast showing the right temporal lesion. B and C: Axial T1-weighted MR images taken on postoperative Day 1 (B) and postoperative month 3 (C). D: Coronal sequences from intraoperative T1-weighted MR images showing the laser probe targeting the right temporal lesion.

\section{Case 3}

A 17-year-old girl with a family history of seizure disorder presented with epilepsy characterized by weekly seizures of staring, incontinence, and automatisms. Brain MRI showed a mesiotemporal cystic lesion measuring 1.0 $\times 0.6 \mathrm{~cm}$ on transverse sections in the left superior aspect of the hippocampus lateral to the midbrain. Laser ablation was delivered to a treatment zone spanning the left hippocampus and amygdala in an anterior to posterior manner. No complications were encountered during the procedure, and postoperative MRI verified ablation of the lesion with minimal involvement of adjacent structures. The patient was seen for follow-up 2 weeks later and was free of neurological deficits on examination; however, she did report 1 seizure since completion of the surgery. At the 6-month follow-up examination with neurology, she reported no new seizures and is now Engel Class I.

\section{Case 4}

This patient was a 12-year-old girl with a history of medically refractory epilepsy with seizures characterized by right-sided clonic activity, hand clapping, staring, and unresponsiveness. These seizures had progressively increased in frequency despite aggressive medical therapy with 6 antiepileptic drugs. Although she had known cortical dysplasia, SPECT studies revealed a potential left temporal seizure origin, and left mesiotemporal sclerosis was identified on brain MRI. Given her atypical seizures and history of dual pathology (cortical dysplasia and mesiotemporal sclerosis), she was offered a craniotomy with temporal lobe resection versus laser ablation of the mesiotemporal sclerosis. Her parents chose laser ablation, favoring less invasive intervention. Using the STarFix platform, the laser probe was introduced into the region of left mesiotemporal sclerosis, and thermal ablation of the amygdala and hippocampus was performed along an anterior to posterior vector. Although her surgery was uncomplicated, she presented 4 days later with decreased coordination and eye pain. Postoperative MRI showed stable changes in the left amygdala and hippocampus consistent with laser ablation. At 6 months since her surgery, she is Engel Class IV, as she has had minimal improvement in her seizure frequency or postoperative symptoms. Her seizures did change in character, however, and are now characterized by stiffening of her face and hyperventilation at night.

\section{Case 5}

This patient was a 36-year-old woman with a history of microprolactinoma currently managed with medications. During interval follow-up for her prolactinoma, she was incidentally found to have a 5-mm nonenhancing left cerebellar lesion on MRI (Fig. 4A). Although she was asymptomatic, close comparison with older MR images suggested a gradual increase in the size of her cerebel- 

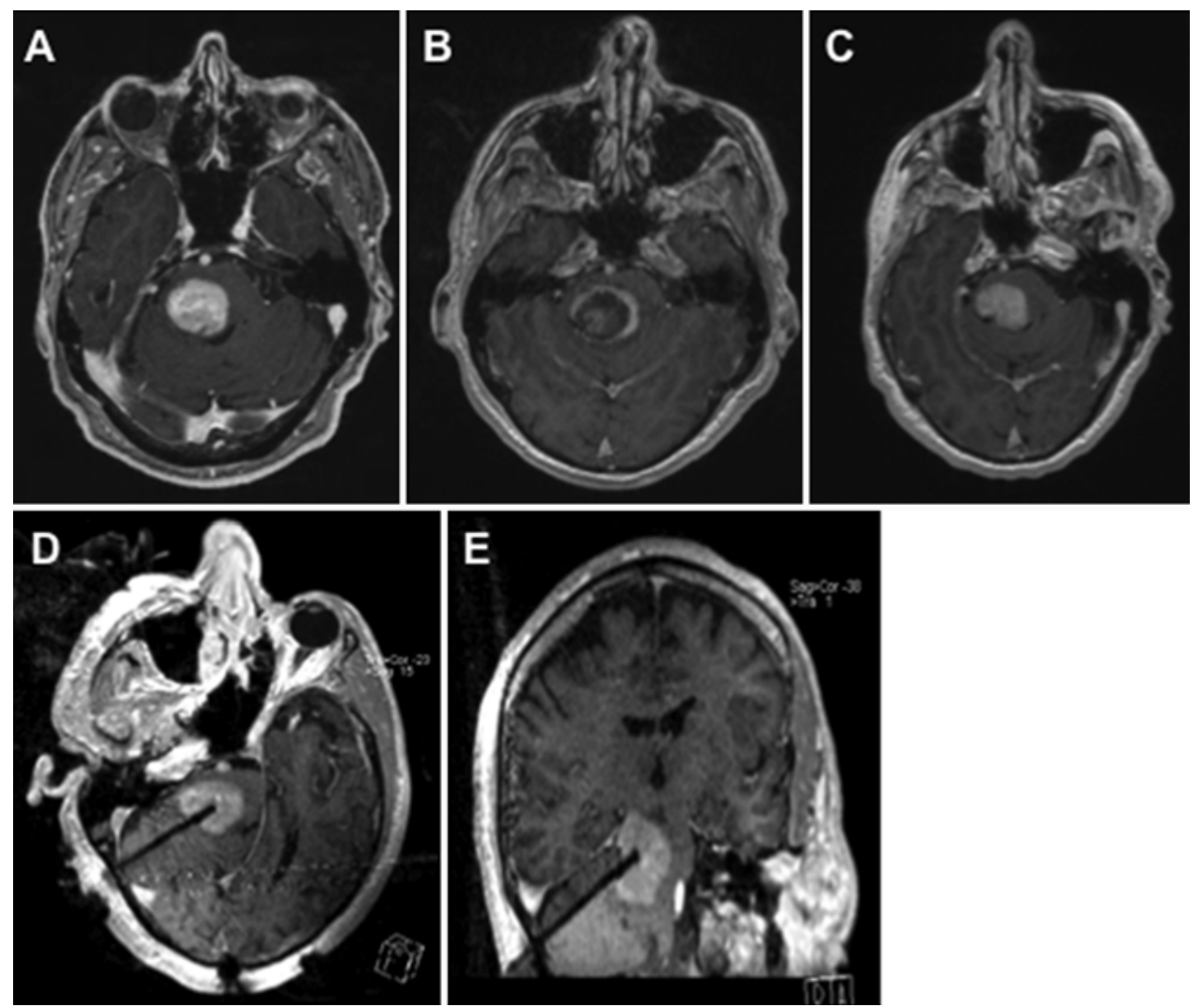

FIG. 3. Brainstem lesion. Magnetic resonance imaging of Case 2 showing a pontine ganglioglioma treated with LITT. A: Preoperative axial T1-weighted MR image with Gd contrast showing the pontine tumor. B and C: Axial T1-weighted MR images obtained on postoperative Day 1 (B) and postoperative month 6 (C). D and E: Axial (D) and coronal (E) intraoperative T1-weighted MR images with contrast showing the tip of the laser probe placed within the pontine tumor.

lar lesion. Use of the STarFix frame facilitated accurate needle biopsy and subsequent laser therapy of the lesion, with minimal residual effect on surrounding tissues as demonstrated by intraoperative (Fig. 4D and E) and postoperative MRI (Fig. 4B and C). After an uncomplicated surgery, the patient was discharged with no neurological deficits and was seen for follow-up 1 month postoperatively with no issues. Pathology revealed the lesion to be a ganglioglioma.

\section{Discussion}

The benefits of LITT in the treatment of difficult-toaccess intracranial lesions have been demonstrated in numerous studies. ${ }^{14,16}$ Real-time guidance of laser ablation with intraoperative MRI has allowed for successful treatment of a variety of lesions including primary and recurrent high-grade gliomas, metastatic disease, and epileptogenic foci. ${ }^{6-8,16}$ More recently, LITT has also been associated with induction of enhanced local permeability of the blood-brain barrier. ${ }^{11}$ This finding demonstrates the expanding potential for LITT to serve in myriad therapeutic strategies, not only in targeting deep-seated brain lesions, but potentially to synergize with medical therapies by enhancing their biodistribution within the brain.

In light of the increasing impact of LITT in the treatment of neurosurgical diseases, addressing the limits of the technology will serve to improve the breadth of its use and applicability. STarFix mT platforms, by simplifying the laser probe insertion process while increasing the accuracy and stability of complex trajectories, can be applied to improve the versatility of LITT. In this report, we demonstrate the feasibility of applying STarFix technology to LITT in a series of 5 patients. Two patients were treated for glial neoplasms, while 3 patients were treated for mesiotemporal epileptogenic foci. For all patients, STarFix $\mathrm{mT}$ platforms were designed and used to guide insertion of the NeuroBlate laser probe. In our limited series, patients tolerated each phase of surgery well, with only transient (and not unexpected) neurological deficits noted in 1 patient (Case 2), and use of the $\mathrm{mT}$ platforms was achieved without difficulty.

We observed treatment effects as evidenced by symptomatic response and changes in enhancement on immediate and late postoperative MRI scans. Imaging findings were consistent with the expected diffusion restriction, coagulative necrosis, and increased peritumoral edema after successful ablation. ${ }^{4}$ In each patient, postablation radiographic findings were well restricted to the original target site.

Currently, there are few competing technologies that may be applied in a similar fashion to LITT. Among these are traditional rigid external stereotactic frames, the Clearpoint SMARTFrame (MRI Interventions), and the ROSA robotic device (Medtech). While these offer highly accurate stereotaxis for probe insertion, ${ }^{10,20}$ STarFix repre- 

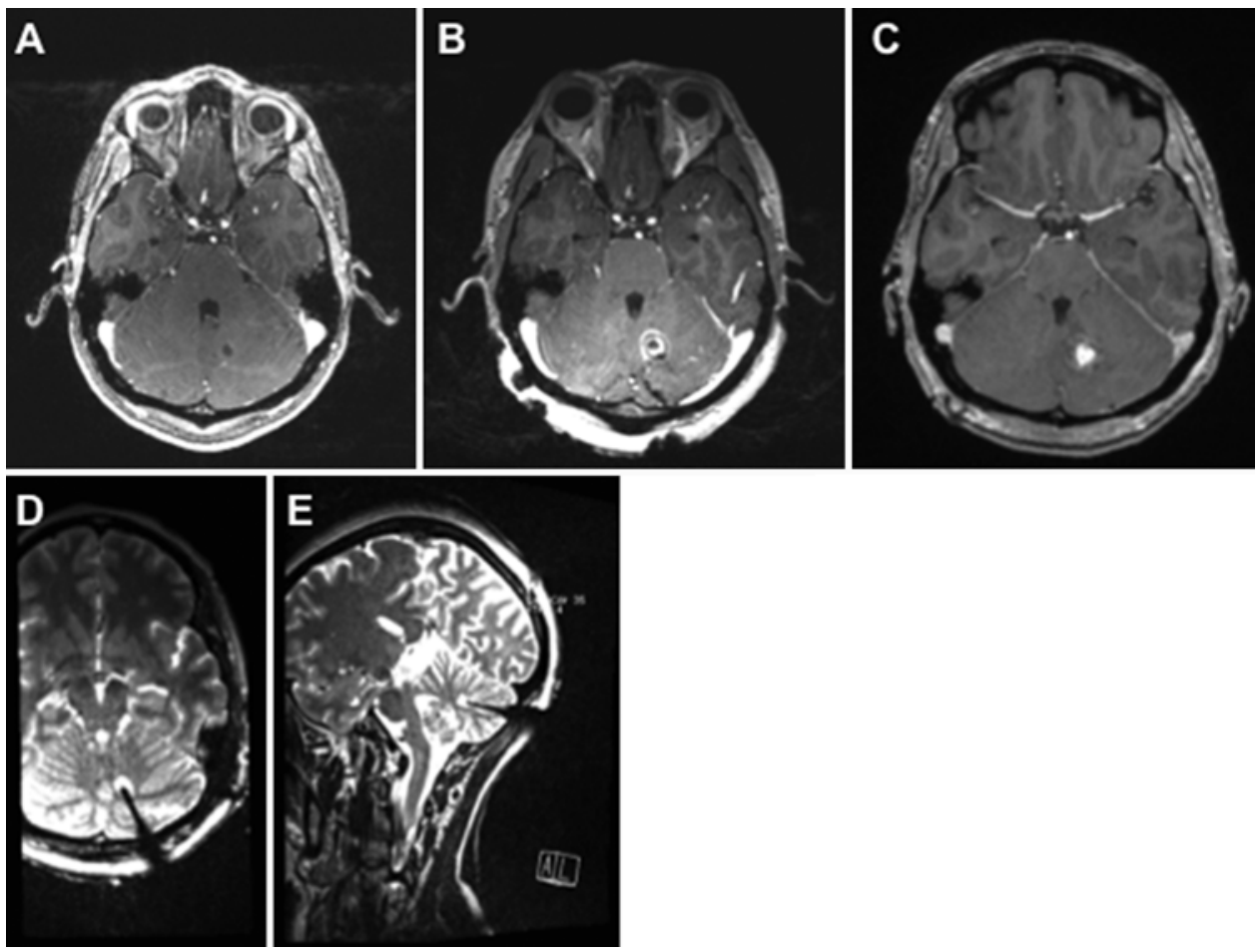

FIG. 4. Cerebellar lesion. Magnetic resonance imaging of Case 5 showing a cerebellar ganglioglioma treated with LITT. A: Preoperative axial T1-weighted MR image with Gd contrast showing a left cerebellar lesion. B: Immediate postoperative axial T1-weighted MR image with contrast showing reactive changes after laser ablation. C: Axial T1-weighted MR image with contrast on postoperative Day 1. D and E: Axial (D) and sagittal (E) intraoperative T1-weighted MR images showing the laser probe positioned within the cerebellar lesion.

sents an easily accessible means of achieving similar levels of accuracy with little to no learning curve. A significant advantage offered by the STarFix design is the ability to anchor the frame at or above the level of the superior nuchal line, where scalp tissue is relatively thin. This allows for facile fixation for targeting the posterior fossa, where the thickness in the overlying soft tissue, including muscle, can complicate the fixation of alternative frames. Because of this reason, the Axiis Stereotactic frame (Monteris) is very challenging to use in the posterior fossa, especially given the requirement to pass screws through the feet of the Axiis base into the skull. LITT trajectories to the posterior fossa and medial temporal lobe are more feasible with the Mini-Bolt (Monteris), but the accuracy of this approach is limited by the stability of the trajectory guide for the various neuronavigation systems, which often cannot withstand the force of a handheld drill.

The accuracy of STarFix platforms has been shown in its applications to DBS, ${ }^{9}$ with STarFix providing equally effective probe implantation when compared with conventional Zamorano-Dujovny frames. ${ }^{12,13}$ When compared with the Nexframe (Medtronic), STarFix has been shown to be more accurate. ${ }^{2}$ Given the similar demands for accuracy and precision required for targeting tumors and epileptogenic foci, we believe that particular LITT cases could benefit from this technology based on our experience with the platform. Our success in incorporating STarFix mT frames into the LITT workflow serves as proof of concept for future endeavors to treat difficult-to-access lesions in critical brain regions.
In addition to accuracy, STarFix allows for the advantageous dissociation of treatment planning from the actual surgery. Indeed, this has been shown to result in shorter operative times for DBS procedures. ${ }^{9}$ In this study, we found that the mean total operative time, accounting for fiducial placement and ablation, trended toward shorter average time, 19 minutes less than our institutional mean for LITT, although this was based on a small sample size and without an appropriately matched control group.

STarFix and the Waypoint Navigation Software are FDA approved, and the applications of these platforms continue to grow. However, disadvantages of the STarFix approach include the need for 2 procedures under general anesthesia within a short time span. Furthermore, the cost associated with the service, STarFix components, and use of the Waypoint Navigator Software may increase the total cost of any given LITT procedure. However, a systematic financial comparison between a standard Mini-Bolt case and a STarFix-based LITT procedure has yet to be performed.

\section{Conclusions}

The continued optimization of intraoperative accuracy of neurosurgical procedures is paramount to improving surgical outcomes. Our experience with the STarFix mT platform demonstrates that novel approaches to stereotactic guidance can be adapted to LITT. With the use of bone fiducials and customized 3D-printed frames, deep lesions within the posterior fossa and medial temporal lobes were 
accurately ablated with minimal postoperative complications. While these results are promising, our study was limited by its inherent retrospective design. The small number of patients we were able to include precludes the possibility of making statistical conclusions on treatment outcomes. Therefore, additional larger studies are needed to fully characterize the impact that personalized stereotactic platforms can have on the therapeutic efficacy of LITT.

\section{References}

1. Balanescu B, Franklin R, Ciurea J, Mindruta I, Rasina A, Bobulescu RC, et al: A personalized stereotactic fixture for implantation of depth electrodes in stereoelectroencephalography. Stereotact Funct Neurosurg 92:117-125, 2014

2. D'Haese PF, Pallavaram S, Konrad PE, Neimat J, Fitzpatrick JM, Dawant BM: Clinical accuracy of a customized stereotactic platform for deep brain stimulation after accounting for brain shift. Stereotact Funct Neurosurg 88:81-87, 2010

3. Ferroli P, Broggi M, Schiavolin S, Acerbi F, Bettamio V, Caldiroli D, et al: Predicting functional impairment in brain tumor surgery: the Big Five and the Milan Complexity Scale. Neurosurg Focus 39(6):E14, 2015

4. Hawasli AH, Bagade S, Shimony JS, Miller-Thomas M, Leuthardt EC: Magnetic resonance imaging-guided focused laser interstitial thermal therapy for intracranial lesions: singleinstitution series. Neurosurgery 73:1007-1017, 2013

5. Hawasli AH, Bandt SK, Hogan RE, Werner N, Leuthardt EC: Laser ablation as treatment strategy for medically refractory dominant insular epilepsy: therapeutic and functional considerations. Stereotact Funct Neurosurg 92:397-404, 2014

6. Hawasli AH, Kim AH, Dunn GP, Tran DD, Leuthardt EC: Stereotactic laser ablation of high-grade gliomas. Neurosurg Focus 37(6):E1, 2014

7. Hawasli AH, Ray WZ, Murphy RKJ, Dacey RG, Leuthardt EC: Magnetic resonance imaging-guided focused laser interstitial thermal therapy for subinsular metastatic adenocarcinoma: technical case report. Neurosurgery 70 (2 Suppl Operative):332-338, 2012

8. Kang JY, Wu C, Tracy J, Lorenzo M, Evans J, Nei M, et al: Laser interstitial thermal therapy for medically intractable mesial temporal lobe epilepsy. Epilepsia 57:325-334, 2016

9. Konrad PE, Neimat JS, Yu H, Kao CC, Remple MS, D'Haese $\mathrm{PF}$, et al: Customized, miniature rapid-prototype stereotactic frames for use in deep brain stimulator surgery: initial clinical methodology and experience from 263 patients from 2002 to 2008. Stereotact Funct Neurosurg 89:34-41, 2011

10. Larson PS, Starr PA, Bates G, Tansey L, Richardson RM, Martin AJ: An optimized system for interventional magnetic resonance imaging-guided stereotactic surgery: preliminary evaluation of targeting accuracy. Neurosurgery 70 (1 Suppl Operative):95-103, 2012

11. Leuthardt EC, Duan C, Kim MJ, Campian JL, Kim AH, Miller-Thomas MM, et al: Hyperthermic laser ablation of recurrent glioblastoma leads to temporary disruption of the peritumoral blood brain barrier. PLoS One 11:e0148613, 2016
12. Matzke C, Hammer N, Weise D, Lindner D, Fritzsch D, Classen J, et al: [Deep brain stimulation using simultaneous stereotactic electrode placement: an alternative to conventional functional stereotaxy?] Nervenarzt 85:1561-1568, 2014 (Ger)

13. Matzke C, Lindner D, Schwarz J, Classen J, Hammer N, Weise D, et al: A comparison of two surgical approaches in functional neurosurgery: individualized versus conventional stereotactic frames. Comput Aided Surg 20:34-40, 2015

14. Missios S, Bekelis K, Barnett GH: Renaissance of laser interstitial thermal ablation. Neurosurg Focus 38(3):E13, 2015

15. Mohammadi AM, Schroeder JL: Laser interstitial thermal therapy in treatment of brain tumors-the NeuroBlate System. Expert Rev Med Devices 11:109-119, 2014

16. Rahmathulla G, Recinos PF, Kamian K, Mohammadi AM, Ahluwalia MS, Barnett GH: MRI-guided laser interstitial thermal therapy in neuro-oncology: a review of its current clinical applications. Oncology 87:67-82, 2014

17. Sharma M, Balasubramanian S, Silva D, Barnett GH, Mohammadi AM: Laser interstitial thermal therapy in the management of brain metastasis and radiation necrosis after radiosurgery: An overview. Expert Rev Neurother 16:223232,2016

18. Stuart RM, Goodman RR: Novel use of a custom stereotactic frame for placement of depth electrodes for epilepsy monitoring. Neurosurg Focus 25(3):E20, 2008

19. Thompson EM, Anderson GJ, Roberts CM, Hunt MA, Selden NR: Skull-fixated fiducial markers improve accuracy in staged frameless stereotactic epilepsy surgery in children. $\mathbf{J}$ Neurosurg Pediatr 7:116-119, 2011

20. Vadera S, Chan A, Lo T, Gill A, Morenkova A, Phielipp NM, et al: Frameless stereotactic robot-assisted subthalamic nucleus deep brain stimulation: case report. World Neurosurg [epub ahead of print], 2015

\section{Disclosures}

Dr. Leuthardt has a consulting relationship with Monteris Medical; he is an owner of Neurolutions, Osteovantage, and Pear Therapeutics. Dr. Chicoine declares research support from IMRIS, Inc., for the study described in this report; he has a consulting relationship with Medtronic, Inc.

\section{Author Contributions}

Conception and design: Kim, Leuthardt. Acquisition of data: all authors. Analysis and interpretation of data: Kim, Dadey, Kamath. Drafting the article: Dadey, Kamath. Critically revising the article: all authors. Reviewed submitted version of manuscript: all authors. Approved the final version of the manuscript on behalf of all authors: Kim. Administrative/technical/material support: Kim, Leuthardt. Study supervision: Kim, Leuthardt.

\section{Correspondence}

Albert H. Kim, Department of Neurological Surgery, Washington University in St. Louis, 660 S Euclid Ave., Box 8057, St. Louis, MO 63110.email: kima@wudosis.wustl.edu. 